\title{
EFFECTS OF PADDLE DIMENSIONS AND BAFFLE CONDITIONS ON THE INTERRELATIONS AMONG DISCHARGE FLOW RATE, MIXING POWER AND MIXING TIME IN MIXING VESSELS
}

\author{
YUJI SANO AND HIROMOTO USUI \\ Department of Chemical Engineering, Yamaguchi University, Ube 755
}

Key Words: Mixing, Discharge Flow Rate, Power Number, Mixing Time, Baffle Condition, Paddle

\begin{abstract}
The interrelations among the discharge flow rate, the mixing power and the mixing time in the fully turbulent range in mixing vessels are investigated experimentally with paddles and baffle plates of various dimensions covering from non-baffled to fully baffled conditions. The discharge flow rate number is correlated as a function of the paddle dimensions and the baffle plate dimensions. The relations between the discharge flow rate number and the power number under incomplete baffle conditions are obtained in two cases. If the baffle condition level is fixed, the power number is proportional to the discharge flow rate number for the various paddles. If the paddle is fixed, the power number is proportional to the square of the discharge flow rate number for various baffle conditions. The mixing time is proportional to the time required to circulate the discharged liquid once, and the number of times of circulation to reach mixing is correlated with paddle dimensions, regardless of baffle conditions.
\end{abstract}

\section{Introduction}

In the performance of mixing vessels there are three important characteristic values: discharge flow rate of an impeller, mixing power and mixing time. Many investigators have reported on the power number and the mixing time ${ }^{6,12)}$ for the effects of impeller dimensions and operating conditions. However, as for the discharge flow rate of impellers, which is an important value for the flow characteristics of the mixing vessel, investigations are rather few and the experimental data are fragmental.

In the previous paper, ${ }^{11\}}$ the authors reported the interrelations of discharge flow rate number $N_{q d}$, power number $N_{p}$ and non-dimensional mixing time $n \theta_{M}$ in the fully developed turbulent range under fully baffled conditions.

In incomplete baffle conditions, however, the effects of baffle plates of various level on the discharge flow rate in connection with the mixing power and the mixing time have not been investigated.

In this work, the interrelations among $N_{q d}, N_{p}$ and $n \theta_{M}$ were investigated experimentally with paddles and baffle plates of various dimensions.

\section{Experimental Apparatus and Procedures}

Measurements were carried out with mixing vessels of diameters $D=0.2 \mathrm{~m}$ and $0.4 \mathrm{~m}$, which were almost the same as used in the previous study. ${ }^{11)}$ The baffle conditions were changed from non-baffled to fully baffled condition by changing baffle width $\left(w_{B} / D=0\right.$,

Received December 22, 1986. Correspondence concerning this article should be addressed to Y. Sano.
$0.025,0.05$ and 0.1$)$ and number $\left(n_{B}=0,2,4,6,8\right.$ and 12). The impellers used were paddles of various sizes $\left(d / D=0.3-0.75, b / D=0.1-0.3, n_{p}=2-6\right)$. The paddle was installed in the axis of the vessel at half the height of liquid depth. Most measurements of the three characteristic parameters $N_{q d}, N_{p}$ and $n \theta_{M}$ were performed in the fully developed turbulent range $\left(\operatorname{Re}>10^{4}\right)$, using water at room temperature. The Reynolds number range for the measurements of $N_{q d}$ and $N_{p}$ was extended on the lower side to $R e=10^{2}$, using glycerol water solution at room temperature. The liquid height was equal to the diameter of the vessel.

The measurement procedures were also the same as described in the previous paper. ${ }^{11)}$ The discharge flow rate was measured by the flow follower method, which was first developed by Marr $\mathrm{Jr}^{4)}$ A piece of coloured absorbent cotton of a few $\mathrm{mm}$ in size was used as the flow follower particle. The impeller power was measured by a torque transducer strain gauge, mounted in the shaft of the impeller. Mixing time was measured by the conductivity method, using two electrode cells made of platinum wire installed at the vessel wall. Changes in difference of resistances of the two electrodes, were recorded by a rapid response recorder. The mixing time was determined by the time at which the signal reached a final value.

\section{Experimental Results and Discussion}

\subsection{Variations of $N_{q d}$ and $N_{p}$ with $R e$}

Illustrations of measurement results of $N_{q d}$ and $N_{p}$ in a wide range of Re are shown in Fig. 1. $N_{q d}$ and $N_{p}$ decrease with increasing $R e$ for the non-baffled con- 


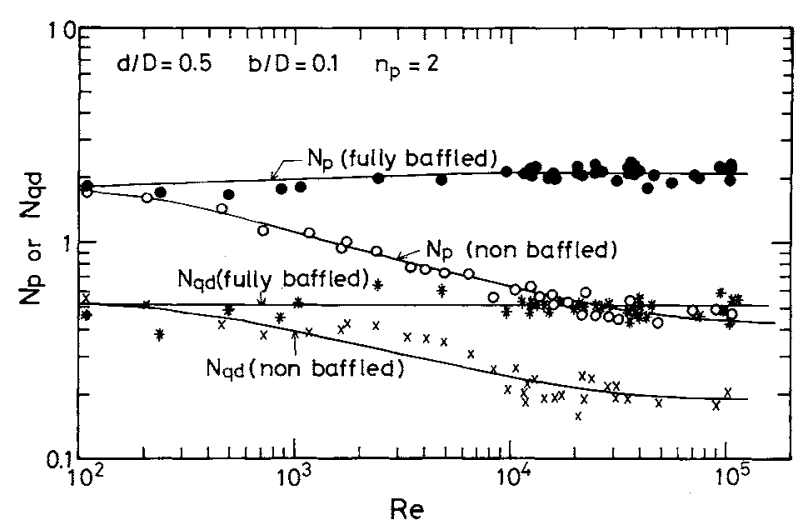

Fig. 1. Variations of $N_{p}$ and $N_{q d}$ with $R e$.

dition, while they remain almost constant for the fully baffled condition. In the fully turbulent range ( $R e>10^{4}$ ), however, both $N_{q d}$ and $N_{p}$ reach constant values even in the non-baffled condition.

\subsection{Fully baffled condition}

Power consumption of the impeller in a mixing vessel is increased by insertion of baffle plates and reaches a maximum value at a certain level of baffle conditions where the tangential flow is suppressed and the cylindrically rotating zone disappears. The fully baffled condition is defined as that at which the power consumption of an impeller reaches a maximum value by insertion of baffle plates on the mixing vessel wall. Nagata ${ }^{6)}$ has shown the fully baffled condition by the following equations.

$$
n_{B} w_{B} / D=0.5
$$

or

$$
n_{B}\left(w_{B} / D\right)^{1.2}=0.35
$$

In this report, the effects of baffle plates are assumed to be evaluated by the term $\alpha=n_{B} w_{B} / D$, which is proportional to the total area of baffle plates.

The effects of baffle plates on $N_{p}, N_{q d}$ and $n \theta_{M}$ for a paddle of $d / D=0.5, b / D=0.1$ and $n_{p}=2$ in the fully turbulent range are shown in Fig. 2 , in which the value of $\alpha$ is changed by various combinations of $n_{B}$ and $w_{B} / D$. The values of $N_{p}$ and $N_{q^{d}}$ are increased and $n \theta_{M}$ is decreased by an increase of $\alpha$. At the value of $\alpha=0.4, N_{p}$ and $N_{q d}$ almost reach the maximum values and $n \theta_{M}$ reaches the minimum value. Then, in this report, the fully baffled condition is defined as follows.

$$
n_{B} w_{B} / D \geq 0.4
$$

Nishikawa et al. ${ }^{9)}$ reported also that the fully baffled condition is attained by $\alpha=0.4$ from the point of view of heat transfer and power consumption in a mixing vessel.

\subsection{Correlation of $N_{q d}$ with impeller dimensions and baffle dimensions}

In the previous paper, ${ }^{11)}$ we reported the correlation

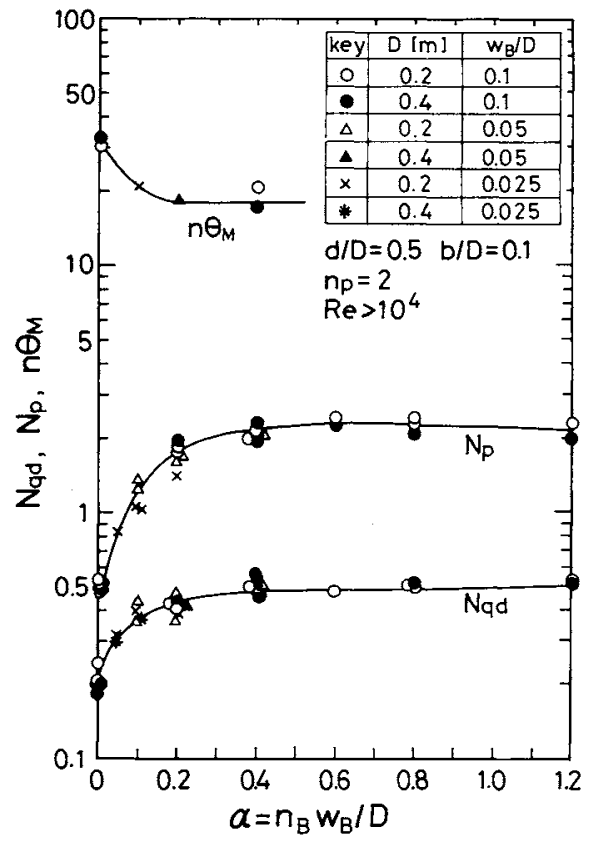

Fig. 2. Determination of fully baffled conditions.

of the discharge flow rate number of paddles in the fully turbulent range $\left(R e>10^{4}\right)$ for the fully baffled condition.

$$
N_{q d F B C}=1.3 \beta_{N_{q d}}
$$

where

$$
\beta_{N_{q d}}=(d / D)^{-0.86}(b / D)^{0.82} n_{p}^{0.60}
$$

It is assumed that the effects of paddle dimensions on $N_{q d}$ in the incomplete baffle condition including nonbaffled condition are the same as in the fully baffled condition, expressed by the term $\beta_{N_{q d}}$. The effects of baffle plates on $N_{q d}$ are considered by the term $\alpha=n_{B} w_{B} / D$.

Figure 3 shows the plot of $N_{q d}$ vs. $\beta_{N_{q d}}$ for both the fully baffled condition and the non-baffled condition. It is noted that both sets of data are fairly well correlated by the term $\beta_{N_{q d}}$, even for the non-baffled condition. The data of incomplete condition of $\alpha=$ $0.05,0.1$ and 0.2 , which include various combinations of $n_{B}$ and $w_{B} / D$, are also well correlated by the term $\beta_{N_{q d}}$, as shown by the lines, where the data were not plotted in order to avoid confusion.

From these plots, the following correlation equation is derived.

$$
N_{q d}=1.3[1-0.62 \exp (-6.8 \alpha)] \beta_{N_{q d}}
$$

in which $\alpha$ is taken as 0.4 if $\alpha \geqq 0.4$. The plots of the correlation of Eq. (6) are shown for all the data in Fig. 4. The average deviation of the correlation is $9.9 \%$. From Eq. (6), the discharge flow rate can be calculated by specifying the dimensions of the paddle and the baffle plate condition. The equation covers the experimental ranges of $(d / D=0.3-0.75, b / D=0.1-0.3$ 


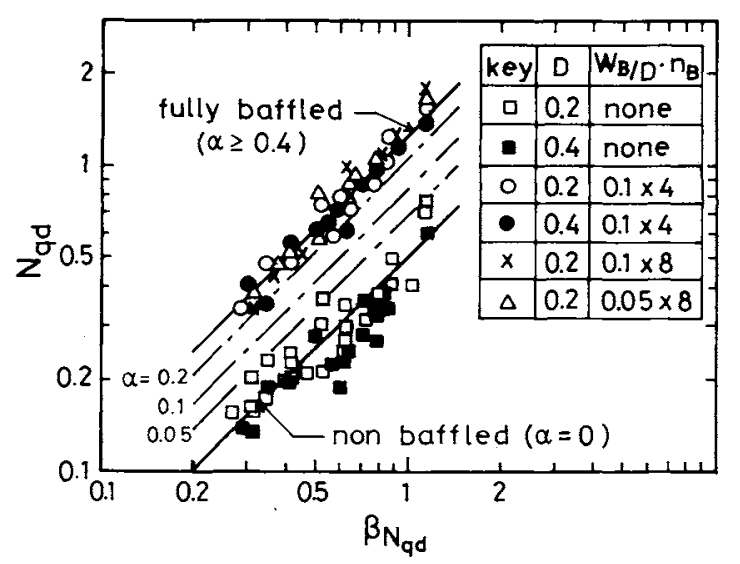

Fig. 3. $N_{q d}$ vs. $\beta_{N q d}$ for fully baffled and non-baffled conditions.

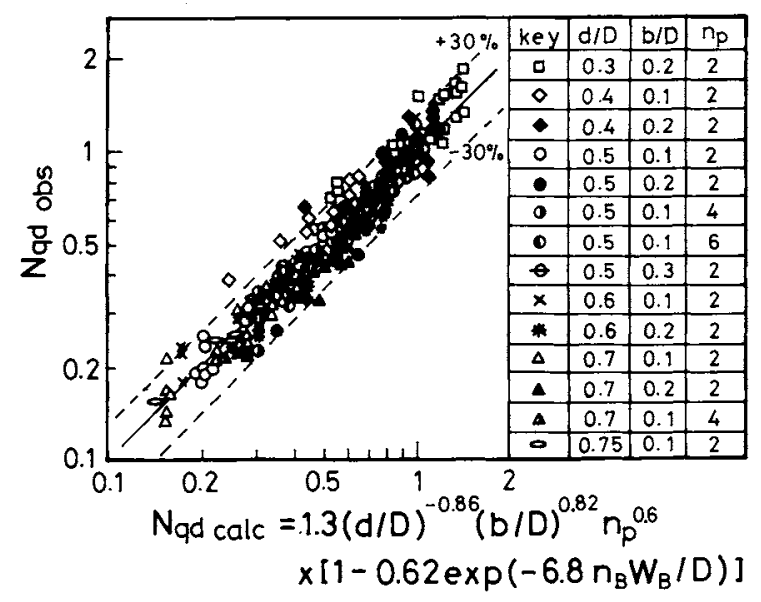

Fig. 4. Correlation of $N_{q d}$ by dimensions of paddles and baffle plates.

and $\left.n_{p}=2-6\right)$ for paddles and $\left(w_{B} / D=0-0.1\right.$ and $n_{B}=$ 1-12) for baffle plates in the fully turbulent range $\left(\operatorname{Re}>10^{4}\right)$.

\subsection{Relation between $N_{q d}$ and $N_{p}$}

In the previous paper, ${ }^{11)}$ the relation between $N_{q d}$ and $N_{p}$ in the fully baffled condition is expressed in the fully developed turbulent range by the following equation, regardless of paddle dimensions.

$$
N_{p}=4.3 N_{q d}^{1,34}
$$

The relation between $N_{q d}$ and $N_{p}$ of the non-baffled condition in the fully turbulent range in which both $N_{p}$ and $N_{q d}$ are almost constant is shown in Fig. 5. From Fig. 5, $N_{p}$ is proportional to $N_{q d}$ regardless of paddle dimensions in the fully turbulent range. The correlation equation for this case is

$$
N_{p}=1.8 N_{q d}
$$

The effects of baffle conditions on the relations between $N_{q d}$ and $N_{p}$ are also evaluated by the term $\alpha=$ $n_{B} w_{B} / D$. In Fig. 6, the relation is shown for $\alpha=0.1$ of various combinations of $n_{B}$ and $w_{B} / D$, for various dimensions of paddles. The relation between $N_{q d}$ and

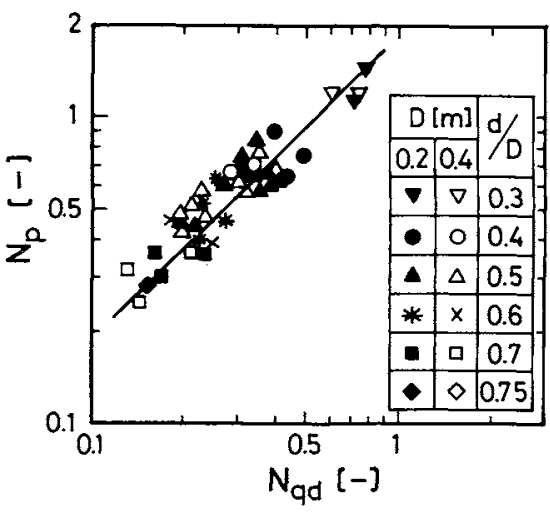

Fig. 5. $N_{p}$ vs. $N_{q d}$ for non-baffled condition.

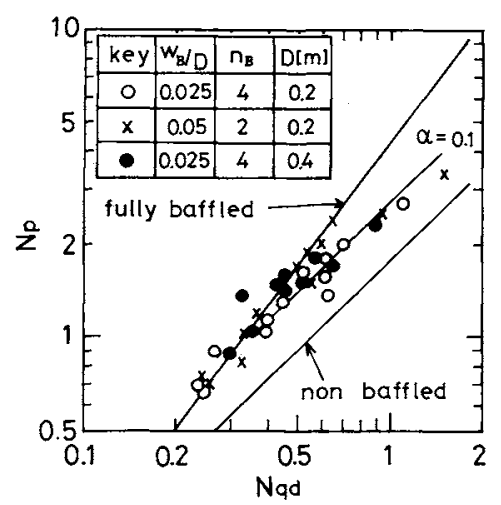

Fig. 6. $N_{p}$ vs. $N_{q d}$ for incomplete baffle conditions of $\alpha=0.1$.

$N_{p}$ is linear with a slope of 1.0 on the right-hand side from the relation for the fully baffled condition, Eq. (7), and fits with Eq. (7) on the left-hand side around the point $N_{q d}=0.3$ which corresponds to the value of $\beta_{N_{q d}}=0.2$. Namely, if $\alpha=0.1$, the relation of $N_{p}$ vs. $N_{q d}$ obeys Eq. (7) for $\beta_{N_{q d}}<0.2$. The same sort of linear relations are obtained for various values of $\alpha$ by various combinations of $n_{B}$ and $w_{B} / D$ as shown in Fig. 7. The correlation for all the data is expressed by the following equation:

$$
N_{p}=4.3[1-0.58 \exp (-6.0 \alpha)] N_{q d}
$$

which is cut off by Eq. (7) for the fully baffled condition as shown in Fig. 7. Then, for $\beta_{N_{q d}}<\beta_{N_{q d c}}$, Eq. (7) is used for the relation of $N_{p}$ vs. $N_{q d}$, where

$$
\beta_{N_{q d c}}=(1 / 1.3)[1-0.58 \exp (-6.0 \alpha)]^{2,94}
$$

For a given impeller, the relations of $N_{q d}$ and $N_{p}$ for various levels of the baffled condition are different from the relations of $N_{q d}$ and $N_{p}$ obtained by changing the dimensions of impellers for a fixed level of baffled condition as described above. Typical plots of $N_{q d}$ and $N_{p}$ of the paddles for various levels of baffled conditions are shown in Fig. 8. The relation between $N_{q d}$ and $N_{p}$ in this Figure is supposed to be linear, with a slope of 2.0. Then, $N_{p}$ is proportional to the square of $N_{q d}$ for the case of a fixed impeller with 


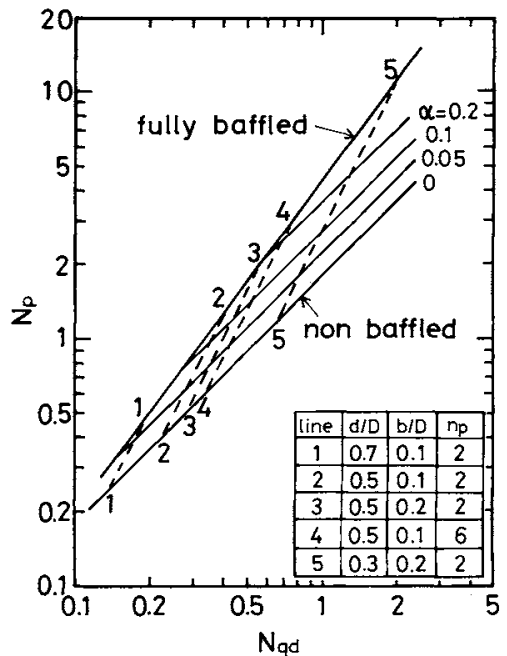

Fig. 7. Summary of relation between $N_{p}$ and $N_{q d}$.

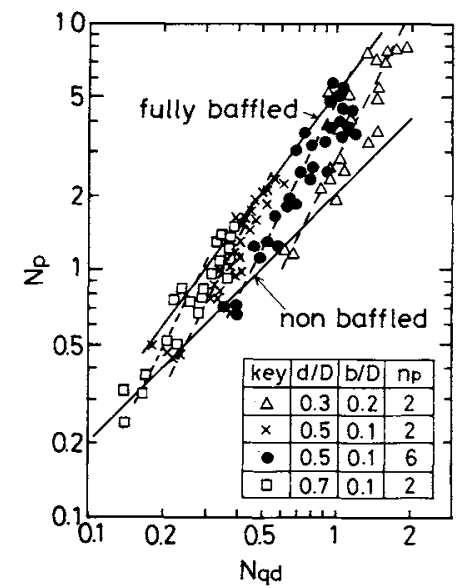

Fig. 8. $N_{p}$ vs. $N_{q d}$ for fixed paddles in various baffle conditions.

various levels of baffled condition. These relations can be expressed from Eqs. (6) and (9), as follows.

$$
N_{p}=3.3 N_{q d}^{2} / \beta_{N_{q d}}
$$

because $[1-0.58 \exp (-6.0 \alpha)] \fallingdotseq[1-0.62 \exp (-6.8 \alpha)]$ for $0 \leq \alpha \leq 0.4$. Equation (11) is limited between Eqs. (7) and (8).

Both Nagase et al..$^{5)}$ and Hiraoka ${ }^{2)}$ have shown the theoretical relationship in which $N_{p}$ is proportional with the square of the discharge flow rate number in a mixing vessel. The linear relation between $N_{p}$ and $N_{q d}$ for the non-baffled condition has also been derived, regardless of impeller dimensions, based on an analogous consideration of the centrifugal pump. ${ }^{12)}$

A summary of the relations between $N_{q d}$ and $N_{p}$ in the present experimental results in the fully turbulent range of $R e>10^{4}$ is shown in Fig. 7. $N_{p}$ is proportional to $N_{q d}$ at a fixed level of baffled condition for various impellers and for a fixed impeller $N_{p}$ is proportional to the square of $N_{q d}$ for various levels of baffled condition. Further investigations will be needed to clarify the behaviour of the relations be- tween $N_{p}$ and $N_{q d}$ found in the present study, in view of the flow pattern in the incomplete baffle condition.

The mechanical efficiency of the mixing system is sometimes defined by the term $N_{q d} / N_{p}{ }^{1)}$ This value is calculated by Eq. (9). The maximum efficiency is obtained for the non-baffled condition as 0.55 . In the baffled condition, the efficiency is always lower than that in the non-baffled condition. As seen in Fig. 7, the efficiency increases when a paddle of larger $(d / D)$, $(b / D)$ and $n_{p}$ is used. In the previous paper, we recommended an impeller of larger $d / D, b / D$ and $n_{p}$ in the fully baffled condition. Here we again recommend a paddle of, say, $d / D=0.75, b / D=0.2$ and $n_{p}=6$, without baffle plates, as a more efficient mixing system.

In the lower range of $R e$, the relations between $N_{p}$ and $N_{q d}$ for the baffle condition level of $\alpha=0.4$ and $\alpha=0$ are shown in Fig. 9. In the lower range of $10^{2}<R e<10^{3}$, the difference between fully baffled and non-baffled conditions becomes smaller and closer relations for $\alpha=0.4$ and $\alpha=0.0$ are obtained, as shown in Fig. 9. However, the proportionality between $N_{p}$ and $N_{q d}$ for $\alpha=0$ seems to hold in this lower $R e$ range.

Figure 10 shows a comparison of relations between $N_{p}$ and $N_{q d}$ in this work with data from the literature, ${ }^{1,7,8,14)}$ in which the discharge flow rates were obtained by direct measurements of radial velocity from impellers. The relationship in the literature for the non-baffled condition $(\alpha=0)^{7,14)}$ deviates upwards from the present results and $N_{p}$ is proportional to $N_{q d}$ with a power of about 0.6 . For the fully baffled condition, the data in the literature scatter. This is due to the difficulty of measurement of radial velocity in three-dimensional flow with high turbulence level in the fully baffled condition.

\subsection{Relation between $\boldsymbol{n} \boldsymbol{\theta}_{M}$ and $\boldsymbol{N}_{q d}$}

In the previous paper, ${ }^{11)}$ we showed that the observed mixing time $\theta_{M}$ can be related to the discharge flow rate by the following equation, assuming that mixing is mainly governed by macro-mixing due to the convection of the discharge flow.

$$
\theta_{M}=m V / q_{d}
$$

For the incomplete baffle condition $(\alpha<0.4)$, the same relation was examined. Typical data of $\theta_{M}$ vs. $V / q_{d}$ are shown in Fig. 11 for an impeller of $d / D=0.5, b / D=$ 0.2 and $n_{p}=\mathbf{2}$. In this figure, the mixing time is proportional to the time required to circulate the discharged liquid, regardless of baffle condition level, from fully baffled to non-baffled, for the given impeller.

The concept that the mixing time is proportional to the average circulation time of discharged liquid in a vessel has been proposed by several investigators. Nagata $^{6)}$ showed that mixing time in the laminar 


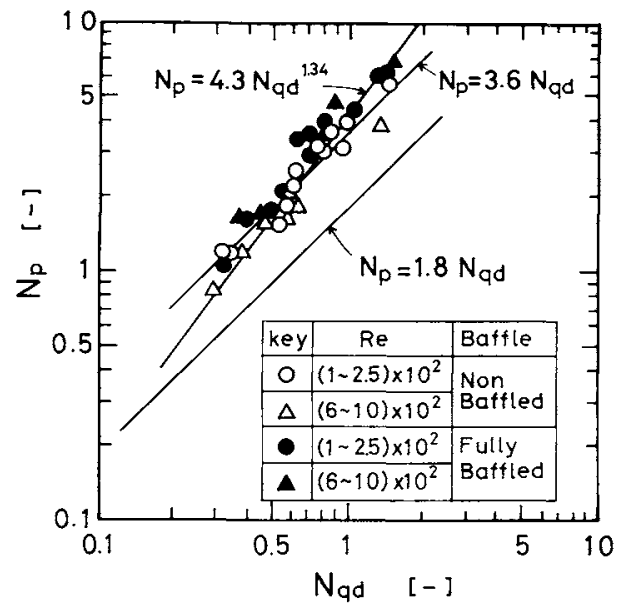

Fig. 9. $N_{p}$ vs. $N_{q d}$ for low Reynolds number

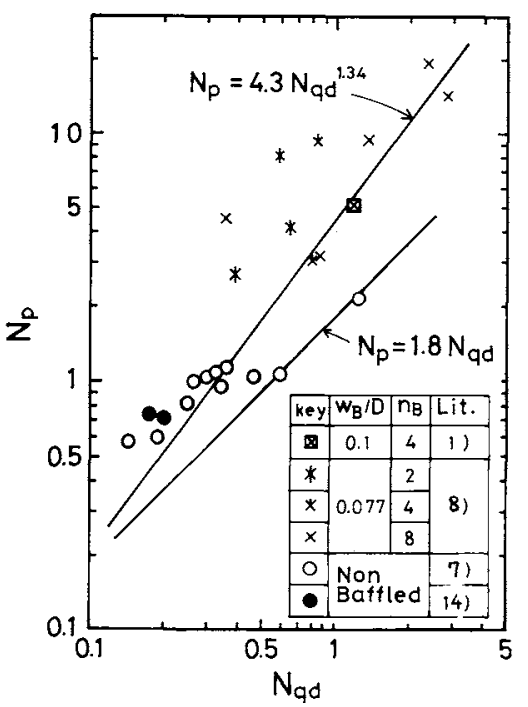

Fig. 10. Comparison of relation between $N_{p}$ and $N_{q d}$ in literature.

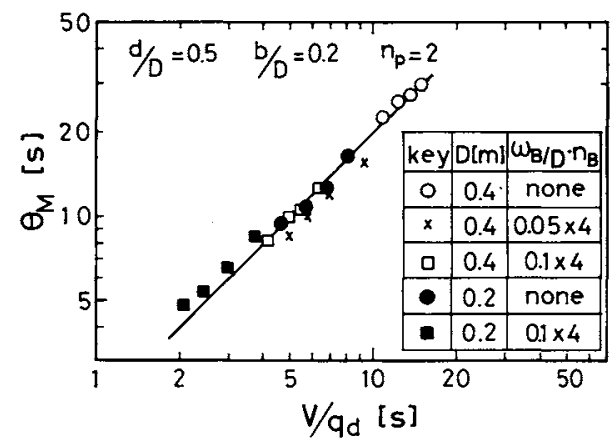

Fig. 11. $\theta_{M}$ vs. $V / q_{d}$ in various baffle conditions.

region is expressed by Eq. (12) and that the value of $m$ is roughly 3.0 irrespective of mixer type, if the mixer is of a circulation type. Van de Vusse ${ }^{13)}$ also reported the effects of impeller dimensions on mixing time based on the concept of Eq. (12) in the turbulent region, demonstrating that the diffusional effect in the turbulent region is so large that mixing time is governed mainly by the pumping action of the impel- ler. Okita and Oyama ${ }^{10)}$ also showed a mixing time correlation based on the concept of Eq. (12) for the mixing of jet flow in a tank. Kamiwano, Yamamoto and Nagata ${ }^{3)}$ derived a correlation equation of mixing time in the fully turbulent range, considering the additive contribution of the effects of both convection and turbulent diffusion to the mixing time. But the additive contribution seems to be questionable, because the mixing time in a vessel is governed by the convection flow even in the laminar flow range as stated by Nagata. ${ }^{6)}$ The present data support the conclusion that mixing time in the fully developed range is mainly governed by the convection effect of the discharge flow from an impeller, given by Eq. (12). Moreover, it is interesting that the ratio $m=\theta_{M} /\left(V / q_{d}\right)$ for a fixed impeller, which means the number of times of circulation of the discharge flow in the vessel to reach the mixing time, is quite independent of baffle conditions. The average value of $m$ for the case of Fig. 11 is 2.0. The average values of $m$ for impellers of various dimensions are obtained from the same plots as in Fig. 11. The average values of $m$ thus obtained are correlated in Fig. 12. The correlation equation is

$$
m=3.8(d / D)^{0.5}\left(n_{p} b / D\right)^{0.1}
$$

which depends only on the dimensions of the paddles and is independent of baffle condition. Equation (13) is the same as the equation derived in the previous paper ${ }^{11)}$ for the fully baffled condition.

From Eqs. (4), (5), (6), (12) and (13), the dimensionless mixing time $n \theta_{M}$ can be expressed as

$$
n \theta_{M}=\left(n \theta_{M}\right)_{F B C} /[1-0.62 \exp (-6.8 \alpha)]
$$

where

$$
\left(n \theta_{M}\right)_{F B C}=2.3(d / D)^{-1.67}(b / D)^{-0.74} n_{p}^{-0.47}
$$

Equation (15) is consistent with the previous results. ${ }^{11)}$

\section{Conclusions}

The discharge flow rate, the mixing power and the mixing time are measured for paddles $(d / D=0.3-0.75$, $b / D=0.1-0.3, n_{p}=2-6$ ), installed at half the height of liquid depth in vessels of $D=0.2 \mathrm{~m}$ and $0.4 \mathrm{~m}$. The baffle conditions are changed in the range $n_{B}=0-12$, $w_{B} / D=0.025-0.1$. The interrelations among the discharge flow rate, the mixing power and the mixing time are obtained in the fully turbulent range. Among these three variables, two are related to one another.

1) The fully baffled condition is defined by Eq. (3).

2) The discharge flow rate is correlated by Eq. (6) for paddles of various dimensions and baffle plates of various dimensions.

3) The relations between $N_{q d}$ and $N_{p}$ are expressed by Eqs. (7), (8) and (9). When the baffle condition is 


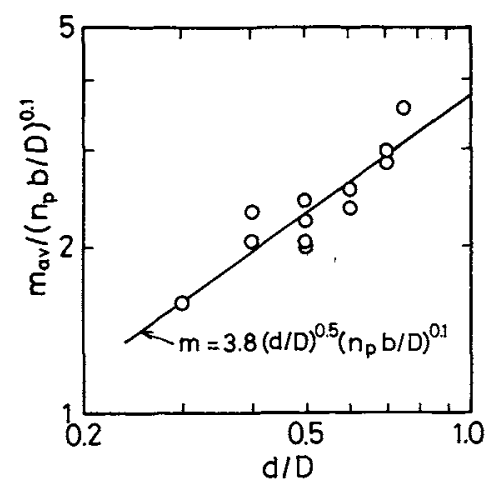

Fig. 12. Correlation of number of times of circulation of discharged liquid to reach mixing time.

fixed, the relations between $N_{q d}$ and $N_{p}$ are linear for the condition of $\beta_{N_{q d}}>\beta_{N_{q d c}}$ where $\beta_{N_{q d c}}$ is given by Eq. (10). When the impeller dimensions are fixed, the relation is $N_{p} \propto N_{q d}^{2}$, as shown by Eq. (11).

4) The mixing time is governed by the convection of the discharge flow as expressed by Eq. (12). The number of times of circulation of the discharged liquid to reach the mixing time is correlated by Eq. (13). The mixing time is correlated with the dimensions of the paddle and the baffle plate, as shown by Eqs. (14) and (15).

5) In the low Reynolds number range, the effects of baffle plates on the relation between $N_{q d}$ and $N_{p}$ become small, as shown in Fig. 9.

\section{Acknowledgment}

The authors appreciate the experimental assistance of student colleagues (Hirohisa Sugiura and Hajime Okido).

$\begin{array}{ll}\text { Nomenclature } & \\ b & =\text { width of impeller blade } \\ D & =\text { diameter of vessel } \\ d & =\text { diameter of impeller } \\ m & =\text { number of times of circulation of discharged } \\ & \text { liquid to reach the mixing time } \\ N_{p} & =\text { power number } P / \rho n^{3} d^{5}\end{array}$

$[\mathrm{m}]$

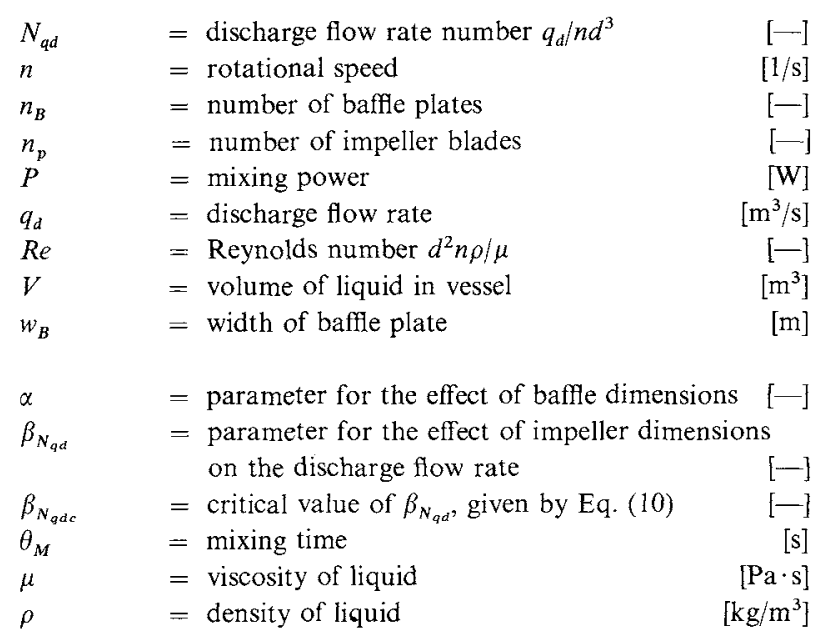

$\langle$ Subscript $\rangle$

$F B C=$ refers to fully baffled condition

\section{Literature Cited}

1) Bertrand, J., J. P. Couderc and H. Angelino: Chem. Eng. Sci., 35, 2157 (1980).

2) Hiraoka, S. and R. Ito: J. Chem. Eng. Japan, 8, 323 (1975).

3) Kamiwano, M., K. Yamamoto and S. Nagata: Kagaku Kōgaku, 31, 365 (1967).

4) Mar, G. R. Jr. and E. F. Johnson: AIChE J., 9, 383 (1963).

5) Nagase, Y., T. Iwamoto, S. Fujita and T. Yoshida: Kagaku Kōgaku, 38, 519 (1974).

6) Nagata, S.: "Mixing. Principles and Applications," p. 24, p. 39 and p. 200, Kodansha; Tokyo, Japan (1975).

7) Nagata, S., K. Yamamoto and M. Ujihara: Kagaku Kōgaku, 23, 130 (1959).

8) Nagata, S., K. Yamamoto, K. Hashimoto and Y. Naruse: Kagaku Kōgaku, 23, 595 (1959).

9) Nishikawa, M., Y. Okamoto, M. Samejima, S. Fujieda and K. Hasimoto: Kagaku Kogaku Ronbunshu, 5, 535 (1979).

10) Okita, N. and Y. Oyama: Kagaku Kögaku, 27, 252 (1963).

11) Sano, Y. and H. Usui: J. Chem. Eng. Japan, 18, 47 (1985).

12) Uhl, V. W. and J. B. Cray: "Mixing Theory and Practice," p. 184, Academic Press, New York (1966).

13) Van de Vusse, J. G.: Chem. Eng. Sci., 4, 178 (1955).

14) Yoshida, T., Y. Nagase, R. Kakumoto, T. Hasegawa and T. Matoba: Kaaku Kógaku, 37, 1038 (1973). 